\title{
PAK4 pathway as a potential therapeutic target in pancreatic cancer
}

\author{
Kiruthikah Thillai ${ }^{*}, 1,2$, Debashis Sarker ${ }^{1,2}$ \& Claire Wells ${ }^{1}$ \\ ${ }^{1}$ Cancer Studies, King's College London, London, SE1 9RT, UK \\ ${ }^{2}$ Department of Medical Oncology, Guy's \& St Thomas' NHS Trust, London, SE1 9RT, UK \\ * Author for correspondence: krith234@gmail.com
}

"PAK4, a serine threonine kinase, has been identified as a potential target in several tumors and its prominence in PDAC suggests it could present an exciting therapeutic opportunity."

First draft submitted: 11 September 2017; Accepted for publication: 22 November 2017; Published online: 6 March 2018

Keywords: p21 activated kinase • PAK4 • pancreatic cancer

Pancreatic ductal adenocarcinoma (PDAC) is a devastating disease and epidemiological trends predict that by 2030 it will be the second leading cause of cancer-related mortality in the USA [1]. Due to the vague symptoms of PDAC, the majority of patients present with advanced disease, which is incurable. Even those with resectable disease are likely to develop a recurrence within 2-3 years. These sobering statistics have fueled the search for more efficacious treatments. Disappointingly, several novel molecularly targeted agents have failed to improve on the modest benefits associated with current chemotherapy regimens. In particular, the use of kinase inhibitors that has revolutionized the management of many solid tumors has yet to make any significant impact in the management of advanced PDAC. However, extensive genomic studies have now begun to unravel the complex genetic environment of PDAC, leading to the discovery of novel molecular aberrations and the hope of new therapeutic strategies are on the horizon. PAK4, a serine threonine kinase, has been identified as a potential target in several tumors and its prominence in PDAC suggests it could present an exciting therapeutic opportunity. This review will discuss the emerging data highlighting the prominence of PAK4 in PDAC and its potential role for transforming patient management.

\section{Pancreatic cancer}

Pancreatic cancer has the worst survival for advanced disease compared with any other common solid malignancy. Even the patients with early-stage disease who undergo extensive surgery are likely to recur within the first 2 years. For many years, the nucleoside analog chemotherapeutic gemcitabine was the treatment of choice for patients with advanced disease. This offered an overall survival of 6 months, with an associated improvement in clinical symptoms. Several large studies searching for an improvement of these outcomes failed until the trial of two novel chemotherapy regimens. The first was FOLFIRNOX and the second was a combination of gemcitabine and nab-paclitaxel $[2,3]$. Both improved the overall survival in patients with advanced disease by 4.3 and 1.8 months, respectively, compared with gemcitabine alone. PDAC has always had worse outcomes with systemic therapy compared with other cancers, a fact partly attributed to the relative chemoresistant nature of the disease. The thick stroma and tumor micro-environment have often been blamed for inadequate drug delivery and the complex genetic signaling for lack of chemotherapy efficacy. Yet noncytotoxic treatments have failed to improve on these modest results with several negative clinical trials, including EGFR, VEGF, BRAF and MMP inhibitors all of which failed to significantly improve overall survival when combined with gemcitabine compared with standard treatment [4-7]. The only positive outcome from a Phase III trial was with the combination of gemcitabine and the anti-EGFR tyrosine kinase inhibitor erlotinib, the benefits of which were minimal and remains the only nonchemotherapeutic agent to be used for this disease [4]. These disappointing results have fueled the search for novel therapeutic targets that could guide future drug development. 


\section{PAKs}

PAKs are a family of serine-threonine kinases that play a role in both oncogenesis and cancer progression. The name arose following their identification as effectors of RhoGTPases (e.g., CDC42 and Rac), each of which is $21 \mathrm{kDa}$ in size. There are two distinct groups: group 1 (Pak1-3) and group 2 (Pak4-6) [8]. Each group is classified by structural and sequence homology and by means of regulation. PAK4 is the best characterized member of the group 2 family and was initially discovered following a search for cytoskeletal regulatory proteins that controlled filopodia formation downstream of CDC42. Following its discovery, several research efforts have sought to interrogate its role in carcinogenesis where it is often overexpressed. PAK4 can mediate cell migration and cell proliferation [9]. There is also evidence that PAK4 regulates the cell cycle and interact with several pertinent oncogenic pathways including the MAPK pathway and the PI3K pathway [10].

\section{PAK4 expression in PDAC}

PAK4 is recurrently overexpressed in PDAC. Comparative genomic hybridization analysis on cDNA microarray of 13 different PDAC cell lines revealed 24 independent amplicons with copy number increases frequently identified at three regions of chromosome 19 (19p13.3, 19q13.1 and 19q13.3) [11]. Deeper analysis confirmed that PAK4 (which occurs at 19q13.1) was frequently amplified correlating with increased copy number variations. In addition, oligonucleotide microarray studies analyzed 92 different PDAC samples, including 22 cell lines, 26 surgical specimens taken form patients with early-stage disease and samples previously published [12]. Several expected genetic amplifications and deletions were identified but focal amplification was found at 19q13 in five patient samples and two cell lines with further immunohistochemical labeling confirming PAK4 was at the 'epicenter' of the region in question. In these seven samples, PAK4 protein expression was increased. Quantitative real time polymerase chain reaction (q-PCR) has also determined that PAK4 expression can be increased in multiple cell lines even in the absence of genetic overexpression. These studies have confirmed that PAK4 is certainly present in a significant proportion of PDAC samples with an increased number demonstrating PAK4 overexpression. These studies have limited patient samples and therefore the true prevalence of PAK4 amplification and copy number variations remain to be determined warranting further deeper analysis of this region in larger PDAC populations.

\section{The role of PAK in PDAC}

PAK4 plays a role in cell adhesion and migration, both essential for the initiation and spread of metastatic disease [13]. In PDAC cells, depletion of PAK4 leads to a reduction in anchorage-independent growth and furthermore activated PAK4 leads to increased migration [9]. Furthermore, PAK4 depletion results change in cell morphology suggesting actin cytoskeletal reorganization and both reduced $2 \mathrm{D}$ migration and $3 \mathrm{D}$ invasion.

PAK4 can interact with both the MAPK and PI3K pathways, which are two canonical pathways downstream of aberrant RAS, an almost universal feature of PDAC tumors [14]. PAK4 has been shown to bind to the p85 component of PI3K and PAK4 depletion leads to reduced phosphorylation of AKT, an important serine-threonine kinase in the PI3K signaling pathway [9]. In addition PAK4 can also lead to reduced ERK activation, part of the MAPK pathway. These findings are significant as several efforts to target the PI3K pathway and MAPK pathway have failed to make a clinical impact in PDAC treatment to date. Alternative strategies to inhibit these pathways are therefore of potential importance and targeting PAK may provide a more effective approach, either as a single agent or in combination.

\section{PAK4: a potential prognostic biomarker}

There have been some small studies suggesting PAK4 expression could predict survival in lung cancer where higher expression was associated with more advanced disease, increased metastatic spread and poorer survival [15]. These findings echo the results from other studies in breast and ovarian cancer [16]. There has been limited data in PDAC, although one small retrospective study surprisingly demonstrated that lower levels of PAK4 expression was associated with worse survival and tumor characteristics [17]. Again, prospective evidence is needed to confirm whether PAK4 could be used as a prognostic biomarker in PDAC.

\section{PAK4: a potential predictive biomarker}

One of the challenges of treating PDAC is the inability to predict which patient may respond to therapy. For effective personalized therapy, unless the 'target' is expressed homogenously, patient selection via predictive biomarkers is vital to identify which patients are most likely to benefit from certain therapies. Therefore, while PAK4 inhibitors 
are developed, parallel work also needs to be done to determine whether PAK4 expression could be used as a predictive tool in determining efficacy of PAK4 inhibitors. Furthermore, one potential role for PAK4 expression would be to guide the use of PI3K inhibitors. As PAK4 expression is correlated with the expression of AKT and simultaneous ERK activity, perhaps PDAC tumors with higher levels of PAK4 would also exhibit increased activity in these pathways [10]. If true, this could allow PAK4 to be a predictive biomarker for drugs targeting these pathways. The lack of predictive biomarkers in clinical trials using tyrosine kinase inhibitors has often been cited as a reason for failure of such drugs. True positive results are diluted in large trials without predetermined biomarker stratification so true responders cannot be identified. More evidence is needed to see whether the expression of PAK4 could be exploited in this way by tissue expression using immunohistochemistry or by genetic sequencing of tumors for copy number variations (CNV) abnormalities.

\section{Direct inhibition of PAK4 in PDAC}

PF-3758309 is an ATP-competitive potent pyranopyrazole inhibitor of PAKs and bind directly to PAK4 with an in vitro potency of 2.7-4.5 $\mathrm{nM}$ [18]. It has been shown to reduce cell proliferation and anchorage-independent growth across a panel of different cell lines including 28 PDAC lines, yet failed to progress past an early-phase study due to unwarranted pharmacokinetic effects. PAK4 allosteric modulators were developed following a small molecule library screen. Three compounds, KPT-9274, KPR-7523 and KPT-7189, target PAK4 [19]. KPT-7189 demonstrated reduced PDAC cell proliferation and inhibition of spheroid formation. Preclinical studies using transgenic mice are ongoing [19]. KPT-9274 and KPT-9307 are both dual NAMPT and PAK4 modulators that can inhibit PDAC cell proliferation. This was recently demonstrated to be through downregulation of Bad phosphorylation and upregulation of miRNAs. KPT-9274 is currently being trialled in an early-phase study (NCT02702492). Furthermore, studies combining PAK4 depletion with traditional chemotherapy have also been encouraging [20]. Three PDAC lines that were resistant to gemcitabine demonstrated reduced cell viability when used in combination with transient PAK4 siRNA, suggesting PAK4 inhibition could be used as in combination with chemotherapy. Inhibition with PAK4-specific drugs remains in the early stages of development but poses a promising treatment strategy worth pursuing.

\section{Conclusion}

Treating PDAC remains a huge challenge. The aggressive biology of the disease leading to the high propensity to metastasis means this highly mutated tumor has the worst survival rates of any solid cancer. Urgent research is needed to develop new therapies with associated biomarker stratification if an improvement is to be seen. PAK4 is an exciting target that could be exploited to treat this disease. Larger studies correlating PAK4 expression and tumor characteristics and survival are needed. Furthermore, results from the development of novel agents inhibiting PAK4 are promising and the preclinical data are awaited with interest, perhaps providing a much needed alternative therapy to this disease.

Financial \& competing interests disclosure

The authors acknowledge financial support from Department of Health via the NIH Research (NIHR) Biomedical Research Centre award to Guy's \& St Thomas' NHS Foundation Trust in partnership with King's College London and King's College Hospital NHS Foundation Trust. King's College London is a UK Experimental Cancer Medicine Centre. $K$ Thillai is the recipient of a Clinical Research Training Fellowship from The Wellcome Trust and a Cancer Research UK training bursary. The views expressed are those of the author(s) and not necessarily those of the NHS, the NIHR or the Department of Health. The authors have no other relevant affiliations or financial involvement with any organization or entity with a financial interest in or financial conflict with the subject matter or materials discussed in the manuscript apart from those disclosed.

No writing assistance was utilized in the production of this manuscript

\section{References}

1. Rahib L, Smith BD, Aizenberg R, Rosenzweig AB, Fleshman JM, Matrisian LM. Projecting cancer incidence and deaths to 2030: the unexpected burden of thyroid, liver, and pancreas cancers in the United States. Cancer Res. 74(11), 2913-2921 (2014).

2. Conroy T, Desseigne F, Ychou M et al. FOLFIRINOX versus gemcitabine for metastatic pancreatic cancer. N. Engl. J. Med. 364(19), 1817-1825 (2011).

3. Von Hoff DD, Ervin T, Arena FP et al. Increased survival in pancreatic cancer with nab-paclitaxel plus gemcitabine. N. Engl. J. Med. 369(18), 1691-1703 (2013). 
4. Moore MJ, Goldstein D, Hamm J et al. Erlotinib plus gemcitabine compared with gemcitabine alone in patients with advanced pancreatic cancer: a Phase III trial of the National Cancer Institute of Canada Clinical Trials Group. J. Clin. Oncol. 25(15), 1960-1966 (2007).

5. Kindler HL, Niedzwiecki D, Hollis D et al. Gemcitabine plus bevacizumab compared with gemcitabine plus placebo in patients with advanced pancreatic cancer: Phase III trial of the Cancer and Leukemia Group B (CALGB 80303). J. Clin. Oncol. 28(22), 3617-3622 (2010).

6. Goncalves A, Gilabert M, Francois E et al. BAYPAN study: a double-blind Phase III randomized trial comparing gemcitabine plus sorafenib and gemcitabine plus placebo in patients with advanced pancreatic cancer. Ann. Oncol. 23(11), 2799-2805 (2012).

7. Bramhall SR, Rosemurgy A, Brown PD, Bowry C, Buckels JA, Marimastat Pancreatic Cancer Study Group. Marimastat as first-line therapy for patients with unresectable pancreatic cancer: a randomized trial. J. Clin. Oncol. 19(15), 3447-3455 (2001).

8. Kumar R, Gururaj AE, Barnes CJ. p21-activated kinases in cancer. Nat. Rev. Cancer 6(6), 459-471 (2006).

9. King H, Thillai K, Whale A et al. PAK4 interacts with p85 alpha: implications for pancreatic cancer cell migration. Sci. Rep. 7, 42575 (2017).

10. Ebi H, Costa C, Faber AC et al. PI3K regulates MEK/ERK signaling in breast cancer via the Rac-GEF, P-Rex1. Proc. Natl Acad. Sci. USA 110(52), 21124-21129 (2013).

11. Mahlamaki EH, Kauraniemi P, Monni O, Wolf M, Hautaniemi S, Kallioniemi A. High-resolution genomic and expression profiling reveals 105 putative amplification target genes in pancreatic cancer. Neoplasia 6(5), 432-439 (2004).

12. Chen S, Auletta T, Dovirak O et al. Copy number alterations in pancreatic cancer identify recurrent PAK4 amplification. Cancer Biol. Ther. 7(11), 1793-1802 (2008).

13. Qu J, Cammarano MS, Shi Q, Ha KC, de Lanerolle P, Minden A. Activated PAK4 regulates cell adhesion and anchorage-independent growth. Mol. Cell Biol. 21(10), 3523-3533 (2001).

14. Wells CM, Abo A, Ridley AJ. PAK4 is activated via PI3K in HGF-stimulated epithelial cells. J. Cell Sci. 115(Pt 20), $3947-3956$ (2002).

15. Cai S, Ye Z, Wang X et al. Overexpression of P21-activated kinase 4 is associated with poor prognosis in non-small cell lung cancer and promotes migration and invasion. J. Exp. Clin. Cancer Res. 34, 48 (2015).

16. He LF, Xu HW, Chen M et al. Activated-PAK4 predicts worse prognosis in breast cancer and promotes tumorigenesis through activation of PI3K/AKT signaling. Oncotarget 8(11), 17573-17585 (2017).

17. Park S, Kim JW, Kim H et al. Prognostic value of p21-activated kinase 4 in resected pancreatic cancer. APMIS 125(8), 699-707 (2017).

18. Murray BW, Guo C, Piraino J et al. Small-molecule p21-activated kinase inhibitor PF-3758309 is a potent inhibitor of oncogenic signaling and tumor growth. Proc. Natl Acad. Sci. USA 107(20), 9446-9451 (2010).

19. Aboukameel A, Muqbil I, Senapedis W et al. Novel p21-activated kinase 4 (PAK4) allosteric modulators overcome drug resistance and stemness in pancreatic ductal adenocarcinoma. Mol. Cancer Ther. 16(1), 76-87 (2017).

20. Moon SU, Kim JW, Sung JH et al. p21-activated kinase 4 (PAK4) as a predictive marker of gemcitabine sensitivity in pancreatic cancer cell lines. Cancer Res. Treat. 47(3), 501-508 (2015). 